\title{
On the Stability of Cubic and Quadratic Mapping in Random Normed Spaces under Arbitrary T-Norms
}

\author{
J. Vahidi, ${ }^{1}$ S. J. Lee, ${ }^{2}$ F. Fallah, ${ }^{3}$ and R. Ahmadi ${ }^{4}$ \\ ${ }^{1}$ Department of Mathematics, Iran University of Science and Technology, Tehran, Iran \\ ${ }^{2}$ Department of Mathematics, Daejin University, Kyeonggi 487-711, Republic of Korea \\ ${ }^{3}$ Department of Mathematics, Mazandaran University of Science and Technology, Behshahr, Iran \\ ${ }^{4}$ Marand Technical College, University of Tabriz, Tabriz, Iran \\ Correspondence should be addressed to S. J. Lee; hyper@daejin.ac.kr
}

Received 15 November 2013; Revised 7 March 2014; Accepted 20 March 2014; Published 30 April 2014

Academic Editor: Zbigniew Leśniak

Copyright (C) 2014 J. Vahidi et al. This is an open access article distributed under the Creative Commons Attribution License, which permits unrestricted use, distribution, and reproduction in any medium, provided the original work is properly cited.

We prove the stability of some functional equations in the random normed spaces under arbitrary $t$-norms.

\section{Introduction and Preliminaries}

The study of stability problems for functional equations is related to a question of Ulam [1] concerning the stability of group homomorphisms and affirmatively answered for Banach spaces by Hyers [2]. Subsequently, the result of Hyers was generalized by Aoki [3] for additive mappings and by Rassias [4] for linear mappings by considering an unbounded Cauchy difference. The paper [4] of Rassias has provided a lot of influence in the development of what we now call HyersUlam-Rassias stability of functional equations. We refer the interested readers for more information on such problems to the papers [5-9]. In addition, some authors investigated the stability in the settings of fuzzy, probabilistic, and random normed spaces, and for more details see [10-22].

The functional equation

$$
f(x+2 y)+f(x-2 y)=4 f(x+y)+4 f(x-y)-6 f(x)
$$

is said to be the cubic functional equation since the function $f(x)=c x^{3}$ is its solution. Every solution of the cubic functional equation is said to be a cubic mapping. The stability problem for the cubic functional equation was solved by Skof [23] for mappings $f: X \rightarrow Y$, where $X$ is a normed space and $Y$ is a Banach space.
The functional equation

$$
\begin{aligned}
f(x+2 y)+f(x-2 y) \\
\quad=4 f(x+y)+4 f(x-y)-6 f(x)+2 f(2 y)-8 f(y)
\end{aligned}
$$

is said to be the quadratic functional equation since the function $f(x)=c x^{2}$ is its solution. Every solution of the quadratic functional equation is said to be a quadratic mapping (see $[8,9]$ ).

Throughout this paper, the space of all probability distribution functions is denoted by $\Delta^{+}=\left\{\begin{array}{lll}F & : R \cup\end{array}\right.$ $\{-\infty,+\infty\} \rightarrow[0,1]: F$ is left-continuous and non decreasing on $R$ and $F(0)=0, F(+\infty)=1\}$ and the subset $D^{+} \subseteq \Delta^{+}$is the set $D^{+}=\left\{F \in \Delta^{+}: l^{-} F(+\infty)=1\right\}$, where $l^{-} f(x)$ denotes the left limit of the function $f$ at the point $x$. The space is partially ordered by the usual pointwise ordering of functions; that is, $F \leqslant G$ if and only if $F(t) \leqslant G(t)$ for all $t$ in $R$. The maximal element for $\Delta^{+}$in this order is the distribution function given by

$$
\varepsilon_{0}(t)= \begin{cases}0 & t \leq 0 \\ 1 & t>0\end{cases}
$$


Definition 1 (see [24]). A mapping $T:[0,1] \times[0,1] \rightarrow$ $[0,1]$ is a continuous triangular norm (briefly, a $t$-norm) if $T$ satisfies the following conditions:

(i) $T$ is commutative and associative;

(ii) $T$ is continuous;

(iii) $T(a, 1)=a$ for all $a \in[0,1]$;

(iv) $T(a, b) \leq T(c, d)$ whenever $a \leq c$ and $b \leq$ $d(a, b, c, d \in[0,1])$.

Recall that, if $T$ is a $t$-norm and $\left\{a_{n}\right\}$ is a given sequence of numbers in [0,1], $T_{i=1}^{n} a_{i}$ is defined recursively by $T_{i=1}^{1} a_{i}=a_{1}$ and $T_{i=1}^{n} a_{i}=T\left(T_{i=1}^{n-1} a_{i}, a_{n}\right)$ for $n \geq 2$.

Definition 2 (see [25]). A random normed space (briefly, RNspace) is a triple $(X, \mu, T)$, where $X$ is a vector space, $T$ is a continuous $t$-norm, and $\mu$ is a mapping from $X$ into $D^{+}$such that, the following conditions hold:

(RN1) $\mu_{x}(t)=\varepsilon_{0}(t)$ for all $t>0$ if and only if $x=0$;

(RN2) $\mu_{\alpha x}(t)=\mu_{x}(t /|\alpha|)$ for all $x \in X, \alpha \neq 0$;

(RN3) $\mu_{x+y}(t+s) \geq T\left(\mu_{x}(t), \mu_{y}(s)\right)$ for all $x, y \in X$ and $t, s \geq 0$.

Definition 3. Let $(X, \mu, T)$ be an $\mathrm{RN}$-space. Consider the following.

(1) A sequence $\left\{x_{n}\right\}$ in $X$ is said to be convergent to $x$ in $X$ if, for every $\epsilon>0$ and $\lambda>0$, there exists a positive integer $N$ such that $\mu_{x_{n}-x}(\epsilon)>1-\lambda$ whenever $n \geq N$.

(2) A sequence $\left\{x_{n}\right\}$ in $X$ is called Cauchy if, for every $\epsilon>0$ and $\lambda>0$, there exists a positive integer $N$ such that $\mu_{x_{n}-x_{m}}(\epsilon)>1-\lambda$ whenever $n \geq m \geq N$.

(3) An $\mathrm{RN}$-space $(X, \mu, T)$ is said to be complete if every Cauchy sequence in $X$ is convergent to a point in $X$.

Theorem 4 (see [24]). If $(X, \mu, T)$ is an RN-space and $\left\{x_{n}\right\}$ is a sequence such that $x_{n} \rightarrow x$, then $\lim _{n \rightarrow \infty} \mu_{x_{n}}(t)=\mu_{x}(t)$ almost everywhere.

\section{On the Stability of Cubic Mappings in $\mathrm{RN}$-Spaces}

Theorem 5. Let $X$ be a normed space, let $\left(R^{+}, \mu^{\prime}, T\right)$ be an $R N-$ space, and let $\varphi: X \times X \rightarrow[0,+\infty)$ be a mapping such that for some $\alpha<1$,

$$
\begin{gathered}
\mu_{\varphi(x, y)}^{\prime}(t) \geq \mu_{8 \alpha \varphi(x / 2, y / 2)}^{\prime}(t) \quad(x, y \in X, t>0) \\
\lim _{m, n \rightarrow \infty} T_{k=1}^{n+1}\left(\mu_{4 \varphi(x, x)}^{\prime}\left(\frac{8 t}{2 \sum_{k=m+1}^{n+m} \alpha^{k-1}}\right)\right. \\
\left.\mu_{\varphi(2 x, x)}^{\prime}\left(\frac{8 t}{2 \sum_{k=m+1}^{n+m} \alpha^{k-1}}\right)\right) \\
=1 \quad(x, y \in X, t>0)
\end{gathered}
$$

and let $(Y, \mu, T)$ be a complete $R N$-space. If $f: X \rightarrow Y$ is an odd mapping such that $f(0)=0$ and

$$
\begin{array}{r}
\mu_{f(x+2 y)+f(x-2 y)-4 f(x+y)-4 f(x-y)+6 f(x)}(t) \geq \mu_{\varphi(x, y)}^{\prime}(t) \\
(x, y \in X, t>0)
\end{array}
$$

then there exists a unique cubic mapping $C: X \rightarrow Y$ such that

$$
\begin{array}{r}
\mu_{f(2 x)-2 f(x)-C(x)}(t) \geq T_{k=1}^{\infty}\left(\mu_{(1 / 2) \varphi(x, x)}^{\prime}\left((1-\alpha) \frac{t}{2}\right),\right. \\
\left.\mu_{(1 / 8) \varphi(2 x, x)}^{\prime}\left((1-\alpha) \frac{t}{2}\right)\right) .
\end{array}
$$

Proof. Letting $x=y$ in (6) we get

$$
\mu_{f(y+2 y)+f(y-2 y)-4 f(y+y)-4 f(y-y)+6 f(y)}(t) \geq \mu_{\varphi(y, y)}^{\prime}(t)
$$

thus

$$
\mu_{f(3 y)+f(-y)-4 f(2 y)-4 f(0)+6 f(y)}(t) \geq \mu_{\varphi(y, y)}^{\prime}(t)
$$

since $f$ is an odd mapping and $f(0)=0$ then we have

$$
\mu_{f(3 y)-4 f(2 y)+5 f(y)}(t) \geq \mu_{\varphi(y, y)}^{\prime}(t) \quad(y \in X, t>0) .
$$

Replacing $x$ by $2 y$ in (6)

$$
\mu_{f(2 y+2 y)+f(2 y-2 y)-4 f(2 y+y)-4 f(2 y-y)+6 f(2 y)}(t) \geq \mu_{\varphi(2 y, y)}^{\prime}(t)
$$

we get

$$
\mu_{f(4 y)-4 f(3 y)+6 f(2 y)-4 f(y)}(t) \geq \mu_{\varphi(2 y, y)}^{\prime}(t)
$$

from Definition 2 we have

$$
\begin{gathered}
\mu_{4 f(3 y)-16 f(2 y)+20 f(y)+f(4 y)-4 f(3 y)+6 f(2 y)-4 f(y)}(t) \\
\geq T\left(\mu_{4 f(3 y)-16 f(2 y)+20 f(y)}\left(\frac{t}{2}\right),\right. \\
\left.\mu_{f(4 y)-4 f(3 y)+6 f(2 y)-4 f(y)}\left(\frac{t}{2}\right)\right) \\
=T\left(\mu_{4(f(3 y)-4 f(2 y)+5 f(y))}\left(\frac{t}{2}\right),\right. \\
\left.\mu_{f(4 y)-4 f(3 y)+6 f(2 y)-4 f(y)}\left(\frac{t}{2}\right)\right) .
\end{gathered}
$$

Therefore by (10) and (12), we get

$$
\begin{aligned}
& \mu_{f(4 y)}-10 f(2 y)+16 f(y)(t) \\
& \geq T\left(\mu_{4 \varphi(y, y)}^{\prime}\left(\frac{t}{2}\right), \mu_{\varphi(2 y, y)}^{\prime}\left(\frac{t}{2}\right)\right) \quad(y \in X, t>0) .
\end{aligned}
$$

Replacing $y$ by $x$ and letting $g(x)=f(2 x)-2 f(x)$ in (14) we get

$$
\begin{aligned}
\mu_{f(4 x)-10 f(2 x)+16 f(x)}(t) & =\mu_{f(4 x)-2 f(2 x)-8 f(2 x)+16 f(x)}(t) \\
& =\mu_{g(2 x)-8 g(x)}(t) .
\end{aligned}
$$


Journal of Function Spaces

3

Then

$$
\mu_{(1 / 8) g(2 x)-g(x)}(t) \geq T\left(\mu_{4 \varphi(x, x)}^{\prime}\left(\frac{8 t}{2}\right), \mu_{\varphi(2 x, x)}^{\prime}\left(\frac{8 t}{2}\right)\right) .
$$

Replacing $x$ by $2^{n} x$ in (16) we obtain

$$
\begin{array}{r}
\mu_{g\left(2^{n+1} x\right) / 8-g\left(2^{n} x\right)}(t) \geq T\left(\mu_{4 \varphi\left(2^{n} x, 2^{n} x\right)}^{\prime}\left(\frac{8 t}{2}\right),\right. \\
\left.\mu_{\varphi\left(2^{n+1} x, 2^{n} x\right)}^{\prime}\left(\frac{8 t}{2}\right)\right),
\end{array}
$$

then

$$
\begin{aligned}
& \mu_{g\left(2^{n+1} x\right) / 8^{n+1}-g\left(2^{n} x\right) / 8^{n}}\left(\frac{t}{8^{n}}\right) \\
& \geq T\left(\mu_{4 \varphi\left(2^{n} x, 2^{n} x\right)}^{\prime}\left(\frac{8 t}{2}\right), \mu_{\varphi\left(2^{n+1} x, 2^{n} x\right)}^{\prime}\left(\frac{8 t}{2}\right)\right) .
\end{aligned}
$$

Whence

$$
\begin{array}{r}
\mu_{g\left(2^{n+1} x\right) / 8^{n+1}-g\left(2^{n} x\right) / 8^{n}}(t) \geq T\left(\mu_{4 \varphi\left(2^{n} x, 2^{n} x\right)}^{\prime}\left(\frac{8^{n+1} t}{2}\right),\right. \\
\left.\mu_{\varphi\left(2^{n+1} x, 2^{n} x\right)}^{\prime}\left(\frac{8^{n+1} t}{2}\right)\right)
\end{array}
$$

and using (4) we have

$$
\begin{array}{r}
\mu_{g\left(2^{n+1} x\right) / 8^{n+1}-g\left(2^{n} x\right) / 8^{n}}(t) \geq T\left(\mu_{8^{n} \alpha^{n} 4 \varphi(x, x)}^{\prime}\left(\frac{8^{n+1} t}{2}\right),\right. \\
\left.\mu_{8^{n} \alpha^{n} \varphi(2 x, x)}^{\prime}\left(\frac{8^{n+1} t}{2}\right)\right) \\
\geq T\left(\mu_{4 \varphi(x, x)}^{\prime}\left(\frac{8^{n+1} t}{2\left(8^{n} \alpha^{n}\right)}\right),\right. \\
\left.\mu_{\varphi(2 x, x)}^{\prime}\left(\frac{8^{n+1} t}{2\left(8^{n} \alpha^{n}\right)}\right)\right)
\end{array}
$$

therefore

$$
\begin{aligned}
& \mu_{g\left(2^{n+1} x\right) / 8^{n+1}-g\left(2^{n} x\right) / 8^{n}}(t) \\
& \geq T\left(\mu_{4 \varphi(x, x)}^{\prime}\left(\frac{8 t}{2 \alpha^{n}}\right), \mu_{\varphi(2 x, x)}^{\prime}\left(\frac{8 t}{2 \alpha^{n}}\right)\right),
\end{aligned}
$$

and from (21) we have

$$
\begin{aligned}
& \mu_{g\left(2^{n+1} x\right) / 8^{n+1}-g\left(2^{n} x\right) / 8^{n}}\left(\frac{\alpha^{n} t}{8}\right) \\
& \geq T\left(\mu_{4 \varphi(x, x)}^{\prime}\left(\frac{t}{2}\right), \mu_{\varphi(2 x, x)}^{\prime}\left(\frac{t}{2}\right)\right),
\end{aligned}
$$

also, from above inequality we have

$$
\begin{gathered}
\mu_{\Sigma_{k=1}^{n} g\left(2^{k} x\right) / 8^{k}-g\left(2^{k-1} x\right) / 8^{k-1}}\left(\sum_{k=1}^{n} \frac{\alpha^{k-1} t}{8}\right) \\
=T\left(\mu_{g(2 x) / 8-g(x)}\left(\frac{t}{8}\right), \mu_{g\left(2^{2} x\right) / 8^{2}-g(2 x) / 8}\left(\frac{\alpha t}{8}\right),\right. \\
\left.\ldots, \mu_{g\left(2^{n} x\right) / 8^{n}-g\left(2^{n-1} x\right) / 8^{n-1}}\left(\frac{\alpha^{n-1} t}{8}\right)\right) \\
=T_{k=1}^{n}\left(\mu_{g\left(2^{k} x\right) / 8^{k}-g\left(2^{k-1} x\right) / 8^{k-1}}\left(\frac{\alpha^{k-1} t}{8}\right)\right) \\
\geq T_{k=1}^{n}\left(T\left(\mu_{4 \varphi(x, x)}^{\prime}\left(\frac{t}{2}\right), \mu_{\varphi(2 x, x)}^{\prime}\left(\frac{t}{2}\right)\right)\right) \\
=T_{k=1}^{n+1}\left(\mu_{4 \varphi(x, x)}^{\prime}\left(\frac{t}{2}\right), \mu_{\varphi(2 x, x)}^{\prime}\left(\frac{t}{2}\right)\right) .
\end{gathered}
$$

Then

$$
\begin{aligned}
& \mu_{g\left(2^{n} x\right) / 8^{n}-g(x)}\left(\sum_{k=1}^{n} \frac{\alpha^{k-1} t}{8}\right) \\
& \geq T_{k=1}^{n+1}\left(\mu_{4 \varphi(x, x)}^{\prime}\left(\frac{t}{2}\right), \mu_{\varphi(2 x, x)}^{\prime}\left(\frac{t}{2}\right)\right), \\
& \mu_{g\left(2^{n} x\right) / 8^{n}-g(x)}(t) \geq T_{k=1}^{n+1}\left(\mu_{4 \varphi(x, x)}^{\prime}\left(\frac{8 t}{2 \sum_{k=1}^{n} \alpha^{k-1}}\right),\right. \\
& \left.\mu_{\varphi(2 x, x)}^{\prime}\left(\frac{8 t}{2 \sum_{k=1}^{n} \alpha^{k-1}}\right)\right) .
\end{aligned}
$$

By replacing $x$ by $2^{m} x$ in (25) we observe that

$$
\begin{array}{r}
\mu_{g\left(2^{n+m} x\right) / 8^{n}-g\left(2^{m} x\right)}(t) \\
\geq T_{k=1}^{n+1}\left(\mu_{4 \varphi\left(2^{m} x, 2^{m} x\right)}^{\prime}\left(\frac{8 t}{2 \sum_{k=1}^{n} \alpha^{k-1}}\right),\right. \\
\left.\mu_{\varphi\left(2^{m+1} x, 2^{m} x\right)}^{\prime}\left(\frac{8 t}{2 \sum_{k=1}^{n} \alpha^{k-1}}\right)\right),
\end{array}
$$

then

$$
\begin{gathered}
\mu_{g\left(2^{n+m} x\right) / 8^{n+m}-g\left(2^{m} x\right) / 8^{m}}\left(\frac{t}{8^{m}}\right) \\
\geq T^{n+1}\left(\mu_{4 \varphi\left(2^{m} x, 2^{m} x\right)}^{\prime}\left(\frac{8 t}{2 \sum_{k=1}^{n} \alpha^{k-1}}\right),\right. \\
\left.\mu_{\varphi\left(2^{m+1} x, 2^{m} x\right)}^{\prime}\left(\frac{8 t}{2 \sum_{k=1}^{n} \alpha^{k-1}}\right)\right), \\
\mu_{g\left(2^{n+m} x\right) / 8^{n+m}-g\left(2^{m} x\right) / 8^{m}}(t) \\
\geq T_{k=1}^{n+1}\left(\mu_{4 \varphi\left(2^{m} x, 2^{m} x\right)}^{\prime}\left(\frac{8^{m+1} t}{2 \sum_{k=1}^{n} \alpha^{k-1}}\right),\right. \\
\left.\mu_{\varphi\left(2^{m+1} x, 2^{m} x\right)}^{\prime}\left(\frac{8^{m+1} t}{2 \sum_{k=1}^{n} \alpha^{k-1}}\right)\right) .
\end{gathered}
$$


Using (4) and Definition 2 we have

$$
\begin{array}{r}
\mu_{g\left(2^{n+m} x\right) / 8^{n+m}-g\left(2^{m} x\right) / 8^{m}}(t) \\
\geq T_{k=1}^{n+1}\left(\mu_{8^{m} \alpha^{m} 4 \varphi(x, x)}^{\prime}\left(\frac{8^{m+1} t}{2 \sum_{k=1}^{n} \alpha^{k-1}}\right),\right. \\
\left.\mu_{8^{m} \alpha^{m} \varphi(2 x, x)}^{\prime}\left(\frac{8^{m+1} t}{2 \sum_{k=1}^{n} \alpha^{k-1}}\right)\right) \\
\geq T_{k=1}^{n+1}\left(\mu_{4 \varphi(x, x)}^{\prime}\left(\frac{8^{m+1} t}{8^{m} \alpha^{m} 2 \sum_{k=1}^{n} \alpha^{k-1}}\right),\right. \\
\left.\mu_{\varphi(2 x, x)}^{\prime}\left(\frac{8^{m+1} t}{8^{m} \alpha^{m} 2 \sum_{k=1}^{n} \alpha^{k-1}}\right)\right) \\
\left.\mu_{\varphi(2 x, x)}^{\prime \prime 1}\left(\frac{8 t}{2 \sum_{4 \varphi(x, x)}^{\prime}\left(\frac{8 t}{2 \sum_{k=m+m}^{n+m} \alpha^{k-1}}\right),}\right)\right) 1,
\end{array}
$$

when $m, n$ tend to $\infty$. Then $\left\{g\left(2^{n} x\right) / 8^{n}\right\}$ is a Cauchy sequence in $(Y, \mu, T)$. Since $(Y, \mu, T)$ is a complete $\mathrm{RN}$-space this sequence converges to some point $C(x) \in Y$. Fix $x \in X$ and put $m=0$ in (28). Then

$$
\begin{array}{r}
\mu_{g\left(2^{n} x\right) / 8^{n}-g(x)}(t) \geq T_{k=1}^{n+1}\left(\mu_{4 \varphi(x, x)}^{\prime}\left(\frac{8 t}{2 \sum_{k=1}^{n} \alpha^{k-1}}\right),\right. \\
\left.\mu_{\varphi(2 x, x)}^{\prime}\left(\frac{8 t}{2 \sum_{k=1}^{n} \alpha^{k-1}}\right)\right)
\end{array}
$$

and from (29) for every $t, \delta>0, x \in X$, we have

$$
\begin{aligned}
& \mu_{C(x)-g(x)}(t+\delta) \\
& =\mu_{C(x)-g\left(2^{n} x\right) / 8^{n}+g\left(2^{n} x\right) / 8^{n}-g(x)}(t+\delta) \\
& \geq T\left(\mu_{C(x)-g\left(2^{n} x\right) / 8^{n}}(\delta), \mu_{g\left(2^{n} x\right) / 8^{n}-g(x)}(t)\right) \\
& \geq T\left(\mu_{C(x)-g\left(2^{n} x\right) / 8^{n}}(\delta),\right. \\
& T_{k=1}^{n+1}\left(\mu_{4 \varphi(x, x)}^{\prime}\left(\frac{8 t}{2 \sum_{k=0}^{n-1} \alpha^{k}}\right),\right. \\
& \left.\left.\mu_{\varphi(2 x, x)}^{\prime}\left(\frac{8 t}{2 \sum_{k=0}^{n-1} \alpha^{k}}\right)\right)\right) .
\end{aligned}
$$

We know when $n$ tend $\infty$, then $g\left(2^{n} x\right) / 8^{n} \rightarrow C(x)$, and $\sum_{k=1}^{n} \alpha^{k-1}=1+\alpha+\alpha^{2}+\cdots+\alpha^{n-1}=\left(1-\alpha^{n}\right) /(1-\alpha) \rightarrow$ $1 /(1-\alpha)$. Therefore taking the $\lim n \rightarrow \infty$ and using (30), and also by using Definition 1(iii) and Definition 2 and (RN2) we get

$$
\begin{array}{r}
\mu_{C(x)-g(x)}(t+\delta) \\
\geq T\left(1, T_{k=1}^{\infty}\left(\mu_{4 \varphi(x, x)}^{\prime}\left(\frac{8(1-\alpha) t}{2}\right),\right.\right. \\
\left.\left.\mu_{\varphi(2 x, x)}^{\prime}\left(\frac{8(1-\alpha) t}{2}\right)\right)\right) \\
=T_{k=1}^{\infty}\left(\mu_{(1 / 2) \varphi(x, x)}^{\prime}\left(\frac{(1-\alpha) t}{2}\right),\right. \\
\left.\mu_{(1 / 8) \varphi(2 x, x)}^{\prime}\left(\frac{(1-\alpha) t}{2}\right)\right)
\end{array}
$$

and since $\delta$ was arbitrary, by taking $\delta \rightarrow 0$ in (31), we have

$$
\begin{array}{r}
\mu_{g(x)-C(x)}(t) \geq T_{k=1}^{\infty}\left(\mu_{(1 / 2) \varphi(x, x)}^{\prime}\left(\frac{(1-\alpha) t}{2}\right),\right. \\
\left.\mu_{(1 / 8) \varphi(2 x, x)}^{\prime}\left(\frac{(1-\alpha) t}{2}\right)\right)
\end{array}
$$

which implies that the Inequality (7) holds. Replacing $x$ and $y$ by $2^{n} x$ and $2^{n} y$ in (6), respectively, we get

$$
\begin{aligned}
& \mu_{f\left(2^{n} x+2^{n+1} y\right)+f\left(2^{n} x-2^{n+1} y\right)-4 f\left(2^{n} x+2^{n} y\right)-4 f\left(2^{n} x-2^{n} y\right)+16 f\left(2^{n} x\right)}(t) \\
& \quad \geq \mu_{\varphi\left(2^{n} x, 2^{n} y\right)}^{\prime}(t),
\end{aligned}
$$

that by division by the $8^{n}$ we have

$\mu_{f\left(2^{n}(x+2 y)\right) / 8^{n}+f\left(2^{n}(x-2 y)\right) / 8^{n}-4 f\left(2^{n}(x+y)\right) / 8^{n}-4 f\left(2^{n}(x-y)\right) / 8^{n}+16 f\left(2^{n} x\right) / 8^{n}}$

$$
\times\left(\frac{t}{8^{n}}\right) \geq \mu_{\varphi\left(2^{n} x, 2^{n} y\right)}^{\prime}(t) .
$$

then

$$
\begin{aligned}
& \mu_{f\left(2^{n}(x+2 y)\right) / 8^{n}+f\left(2^{n}(x-2 y)\right) / 8^{n}-4 f\left(2^{n}(x+y)\right) / 8^{n}-4 f\left(2^{n}(x-y)\right) / 8^{n}+16 f\left(2^{n} x\right) / 8^{n}}^{\prime} \\
& \times(t) \geq \mu_{\varphi\left(2^{n} x, 2^{n} y\right)}^{\prime}\left(8^{n} t\right) \geq \mu_{8^{n} \alpha^{n} \varphi(x, y)}^{\prime}\left(8^{n} t\right) \geq \mu_{\varphi(x, y)}^{\prime}\left(\frac{t}{\alpha^{n}}\right)
\end{aligned}
$$

for all $x, y \in X$ and for all $t>0$. Since $\lim _{n \rightarrow \infty} \mu_{\varphi(x, y)}^{\prime}\left(t / \alpha^{n}\right)=$ 1 , we conclude that $C$ fulfills (1). To prove the uniqueness of the cubic function $C$, let us assume that there exists a quadratic function $D: X \rightarrow Y$ which satisfies (7). Obviously 
we have $C\left(2^{n} x\right)=8^{n} C(x)$ and $D\left(2^{n} x\right)=8^{n} D(x)$ for all $x \in X$ and $n \in \mathbb{N}$. It follows from (7) and (32) that for each $x \in X$,

$$
\begin{gathered}
\mu_{C(x)-D(x)}(t) \\
\geq \mu_{C\left(2^{n} x\right)-D\left(2^{n} x\right)}\left(8^{n} t\right) \\
\geq T\left(\mu_{C\left(2^{n} x\right)-g\left(2^{n} x\right)}\left(\frac{8^{n} t}{2}\right),\right. \\
\left.\mu_{g\left(2^{n} x\right)-D\left(2^{n} x\right)}\left(\frac{8^{n} t}{2}\right)\right) \\
\geq T\left(1, T_{k=1}^{\infty}\left(\mu_{(1 / 2) \varphi\left(2^{n} x, 2^{n} x\right)}^{\prime}\left((1-\alpha) \frac{8^{n} t}{2}\right),\right.\right. \\
\left.\left.\mu_{(1 / 8) \varphi\left(2^{n+1} x, 2^{n} x\right)}^{\prime}\left((1-\alpha) \frac{8^{n} t}{2}\right)\right)\right) \\
\geq T_{k=1}^{\infty}\left(\mu_{\left(8^{n} \alpha^{n} / 2\right) \varphi(x, x)}^{\prime}\left((1-\alpha) \frac{8^{n} t}{2}\right),\right. \\
\left.\mu_{\left(8^{n} \alpha^{n} / 8\right) \varphi(2 x, x)}^{\prime}\left((1-\alpha) \frac{8^{n} t}{2}\right)\right) \\
\left.\mu_{(1 / 8) \varphi(2 x, x)}^{\prime}\left((1-\alpha) \frac{t}{2 \alpha^{n}}\right)\right) \longrightarrow 1,
\end{gathered}
$$

then $\mu_{C(x)-D(x)}(t)=1$, for all $t>0$. And so $C(x)=D(x)$. This completes the proof.

\section{On the Stability of Quadratic Mappings in $\mathbf{R N}$-Spaces}

Theorem 6. Let $X$ be a normed space, let $\left(R^{+}, \mu^{\prime}, T\right)$ be an $R N$ space, and let $\varphi: X \times X \rightarrow[0,+\infty)$ be a mapping such that for some $\alpha<1$,

$$
\begin{array}{r}
\mu_{\varphi(x, y)}^{\prime}(t) \geq \mu_{4 \alpha \varphi(x / 2, y / 2)}^{\prime}(t) \quad(x, y \in X, t>0), \\
\lim _{m, n \rightarrow \infty} T_{k=1}^{n+1}\left(\mu_{4 \varphi(x, x)}^{\prime}\left(\frac{4 t}{2 \sum_{j=0}^{n+m-1} \alpha^{j}}\right)\right. \\
\left.\mu_{\varphi(2 x, x)}^{\prime}\left(\frac{4 t}{2 \sum_{j=0}^{n+m-1} \alpha^{j}}\right)\right)=1 \\
(x, y \in X, t>0)
\end{array}
$$

and let $(Y, \mu, T)$ be a complete $R N$-space. If $f: X \rightarrow Y$ is an even mapping such that $f(0)=0$ and

$$
\mu_{f(x+2 y)+f(x-2 y)-4 f(x+y)-4 f(x-y)+6 f(x)-2 f(2 y)+8 f(y)}(t) \geq \mu_{\varphi(x, y)}^{\prime}(t) \quad(x, y \in X, t>0),
$$

then there exists a unique quadratic mapping $Q: X \rightarrow Y$ such that

$$
\begin{aligned}
& \mu_{f(2 x)-16 f(x)-\mathrm{Q}(x)}(t) \\
& \quad \geq T_{k=1}^{\infty}\left(\mu_{(1 / 2) \varphi(x, x)}^{\prime}\left(\frac{(1-\alpha) t}{2}\right), \mu_{(1 / 4) \varphi(2 x, x)}^{\prime}\left(\frac{(1-\alpha) t}{2}\right)\right) .
\end{aligned}
$$

Proof. Letting $x=y$ in (38) we get

$$
\mu_{f(3 y)+f(-y)-4 f(2 y)-4 f(0)+6 f(y)-2 f(2 y)+8 f(y)}(t) \geq \mu_{\varphi(y, y)}^{\prime}(t) .
$$

Since $f$ is an even mapping and $f(0)=0$ then we have

$$
\mu_{f(3 y)-6 f(2 y)+15 f(y)}(t) \geq \mu_{\varphi(y, y)}^{\prime}(t) \quad(y \in X, t>0) .
$$

Replacing $x$ by $2 y$ in (38) we get

$$
\mu_{f(4 y)-4 f(3 y)+4 f(2 y)+4 f(y)}(t) \geq \mu_{\varphi(2 y, y)}^{\prime}(t)
$$

and by (41) and (42), we get

$$
\begin{aligned}
& \mu_{f(4 y)-20 f(2 y)+64 f(y)}(t) \\
& \geq T\left(\mu_{4 f(3 y)-24 f(2 y)+60 f(y)}\left(\frac{t}{2}\right),\right. \\
& \left.\mu_{f(4 y)-4 f(3 y)+4 f(2 y)+4 f(y)}\left(\frac{t}{2}\right)\right) \\
& =T\left(\mu_{4(f(3 y)-6 f(2 y)+15 f(y))}\left(\frac{t}{2}\right),\right. \\
& \left.\mu_{f(4 y)-4 f(3 y)+4 f(2 y)+4 f(y)}\left(\frac{t}{2}\right)\right) .
\end{aligned}
$$

Therefore

$$
\begin{array}{r}
\mu_{f(4 y)-20 f(2 y)+64 f(y)}(t) \geq T\left(\mu_{4 \varphi(y, y)}^{\prime}\left(\frac{t}{2}\right), \mu_{\varphi(2 y, y)}^{\prime}\left(\frac{t}{2}\right)\right) \\
(y \in X, t>0) .
\end{array}
$$


Replacing $y$ by $x$ and letting $g(x)=f(2 x)-16 f(x)$ in (44), we get

$$
\begin{aligned}
& \mu_{f(4 x)-20 f(2 x)+64 f(x)}(t) \\
& \quad \geq T\left(\mu_{4 \varphi(x, x)}^{\prime}\left(\frac{t}{2}\right), \mu_{\varphi(2 x, x)}^{\prime}\left(\frac{t}{2}\right)\right), \\
& \mu_{f(4 x)-20 f(2 x)+64 f(x)}(t) \\
& \quad=\mu_{f(4 x)-16 f(2 x)-4 f(2 x)+64 f(x)}(t) \\
& =\mu_{g(2 x)-4 g(x)}(t) .
\end{aligned}
$$

Then

$$
\mu_{g(2 x)-4 g(x)}(t) \geq T\left(\mu_{4 \varphi(x, x)}^{\prime}\left(\frac{t}{2}\right), \mu_{\varphi(2 x, x)}^{\prime}\left(\frac{t}{2}\right)\right)
$$

that is,

$$
\mu_{(1 / 4) g(2 x)-g(x)}(t) \geq T\left(\mu_{4 \varphi(x, x)}^{\prime}\left(\frac{4 t}{2}\right), \mu_{\varphi(2 x, x)}^{\prime}\left(\frac{4 t}{2}\right)\right) .
$$

Replacing $x$ by $2^{n} x$ in (46) we obtain

$$
\begin{aligned}
& \mu_{g\left(2^{n+1} x\right) / 4-g\left(2^{n} x\right)}(t) \\
& \quad \geq T\left(\mu_{4 \varphi\left(2^{n} x, 2^{n} x\right)}^{\prime}\left(\frac{4 t}{2}\right), \mu_{\varphi\left(2^{n+1} x, 2^{n} x\right)}^{\prime}\left(\frac{4 t}{2}\right)\right),
\end{aligned}
$$

then

$$
\begin{aligned}
& \mu_{g\left(2^{n+1} x\right) / 4^{n+1}-g\left(2^{n} x\right) / 4^{n}}\left(\frac{t}{4^{n}}\right) \\
& \quad \geq T\left(\mu_{4 \varphi\left(2^{n} x, 2^{n} x\right)}^{\prime}\left(\frac{4 t}{2}\right), \mu_{\varphi\left(2^{n+1} x, 2^{n} x\right)}^{\prime}\left(\frac{4 t}{2}\right)\right) .
\end{aligned}
$$

By using (2) and (37) we have

$$
\begin{gathered}
\mu_{g\left(2^{n+1} x\right) / 4^{n+1}-g\left(2^{n} x\right) / 4^{n}}(t) \\
\geq T\left(\mu_{4 \varphi\left(2^{n} x, 2^{n} x\right)}^{\prime}\left(\frac{4^{n+1} t}{2}\right),\right. \\
\left.\mu_{\varphi\left(2^{n+1} x, 2^{n} x\right)}^{\prime}\left(\frac{4^{n+1} t}{2}\right)\right), \\
\mu_{g\left(2^{n+1} x\right) / 4^{n+1}-g\left(2^{n} x\right) / 4^{n}(t)}\left(\mu_{4^{n} \alpha^{n} 4 \varphi(x, x)}^{\prime}\left(\frac{4^{n+1} t}{2}\right),\right. \\
\left.\mu_{4^{n} \alpha^{n} \varphi(2 x, x)}^{\prime}\left(\frac{4^{n+1} t}{2}\right)\right) \\
\geq T\left(\mu_{4 \varphi(x, x)}^{\prime}\left(\frac{4^{n+1} t}{2\left(4^{n} \alpha^{n}\right)}\right),\right. \\
\left.\mu_{\varphi(2 x, x)}^{\prime}\left(\frac{4^{n+1} t}{2\left(4^{n} \alpha^{n}\right)}\right)\right)
\end{gathered}
$$

so

$$
\begin{array}{r}
\mu_{g\left(2^{n+1} x\right) / 4^{n+1}-g\left(2^{n} x\right) / 4^{n}}(t) \\
\geq T\left(\mu_{4 \varphi(x, x)}^{\prime}\left(\frac{4 t}{2 \alpha^{n}}\right),\right. \\
\left.\mu_{\varphi(2 x, x)}^{\prime}\left(\frac{4 t}{2 \alpha^{n}}\right)\right),
\end{array}
$$

and from (51) we have

$$
\begin{aligned}
& \mu_{g\left(2^{n+1} x\right) / 4^{n+1}-g\left(2^{n} x\right) / 4^{n}}\left(\frac{\alpha^{n} t}{4}\right) \\
& \geq T\left(\mu_{4 \varphi(x, x)}^{\prime}\left(\frac{t}{2}\right), \mu_{\varphi(2 x, x)}^{\prime}\left(\frac{t}{2}\right)\right),
\end{aligned}
$$

and also, from the above inequality we have,

$$
\begin{gathered}
\mu_{\sum_{k=1}^{n} g\left(2^{k} x\right) / 4^{k}-g\left(2^{k-1} x\right) / 4^{k-1}}\left(\sum_{k=1}^{n} \frac{\alpha^{k-1} t}{4}\right) \\
=T_{k=1}^{n}\left(\mu_{g(2 x) / 4-g(x)}\left(\frac{t}{4}\right), \mu_{g\left(2^{2} x\right) / 4^{2}-g(2 x) / 4}\left(\frac{\alpha t}{4}\right),\right. \\
\left.\quad \ldots, \mu_{g\left(2^{n} x\right) / 4^{n}-g\left(2^{n-1} x\right) / 4^{n-1}}\left(\frac{\alpha^{n-1} t}{4}\right)\right) \\
\geq T_{k=1}^{n}\left(\mu_{g\left(2^{k} x\right) / 4^{k}-g\left(2^{k-1} x\right) / 4^{k-1}}\left(\frac{\alpha^{k-1} t}{4}\right)\right) \\
=T_{k=1}^{n}\left(T\left(\mu_{4 \varphi(x, x)}^{\prime}\left(\frac{t}{2}\right), \mu_{\varphi(2 x, x)}^{\prime}\left(\frac{t}{2}\right)\right)\right) \\
T_{k=1}^{n+1}\left(\mu_{4 \varphi(x, x)}^{\prime}\left(\frac{t}{2}\right), \mu_{\varphi(2 x, x)}^{\prime}\left(\frac{t}{2}\right)\right) .
\end{gathered}
$$

Hence

$$
\begin{array}{r}
\mu_{g\left(2^{n} x\right) / 4^{n}-g(x)}(t) \\
\geq T_{k=1}^{n+1}\left(\mu_{4 \varphi(x, x)}^{\prime}\left(\frac{4 t}{2 \sum_{k=1}^{n} \alpha^{k-1}}\right),\right. \\
\left.\mu_{\varphi(2 x, x)}^{\prime}\left(\frac{4 t}{2 \sum_{k=1}^{n} \alpha^{k-1}}\right)\right) .
\end{array}
$$

By replacing $x$ by $2^{m} x$ in (54) we obtain

$$
\begin{array}{r}
\mu_{g\left(2^{n+m} x\right) / 8^{n}-g\left(2^{m} x\right)}(t) \\
\geq T_{k=1}^{n+1}\left(\mu_{4 \varphi\left(2^{m} x, 2^{m} x\right)}^{\prime}\left(\frac{4 t}{2 \sum_{j=0}^{n-1} \alpha^{j}}\right),\right. \\
\left.\mu_{\varphi\left(2^{m+1} x, 2^{m} x\right)}^{\prime}\left(\frac{4 t}{2 \sum_{j=0}^{n-1} \alpha^{j}}\right)\right),
\end{array}
$$


then

$$
\begin{array}{r}
\mu_{g\left(2^{n+m} x\right) / 4^{n+m}-g\left(2^{m} x\right) / 4^{m}}\left(\frac{t}{4^{m}}\right) \\
\geq T_{k=1}^{n+1}\left(\mu_{4 \varphi\left(2^{m} x, 2^{m} x\right)}^{\prime}\left(\frac{4 t}{2 \sum_{j=0}^{n-1} \alpha^{j}}\right),\right. \\
\left.\mu_{\varphi\left(2^{m+1} x, 2^{m} x\right)}^{\prime}\left(\frac{4 t}{2 \sum_{j=0}^{n-1} \alpha^{j}}\right)\right), \\
\mu_{g\left(2^{n+m} x\right) / 4^{n+m}-g\left(2^{m} x\right) / 4^{m}(t)}\left(\frac{4^{m+1} t}{2 \sum_{j=0}^{n-1} \alpha^{j}}\right), \\
\left.\mu_{\varphi\left(2^{m+1} x, 2^{m} x\right)}^{\prime}\left(\frac{4^{m+1} t}{2 \sum_{j=0}^{n-1} \alpha^{j}}\right)\right) .
\end{array}
$$

Using (37) and Definition 2 we observe that

$$
\begin{gathered}
\mu_{g\left(2^{n+m} x\right) / 4^{n+m}-g\left(2^{m} x\right) / 4^{m}}(t) \\
\geq T_{k=1}^{n+1}\left(\mu_{4^{m} \alpha^{m} 4 \varphi(x, x)}^{\prime}\left(\frac{4^{m+1} t}{2 \sum_{j=0}^{n-1} \alpha^{j}}\right),\right. \\
\left.\mu_{4^{m} \alpha^{m} \varphi(2 x, x)}^{\prime}\left(\frac{4^{m+1} t}{2 \sum_{j=0}^{n-1} \alpha^{j}}\right)\right) \\
\geq T_{k=1}^{n+1}\left(\mu_{4 \varphi(x, x)}^{\prime}\left(\frac{4^{m+1} t}{4^{m} \alpha^{m} 2 \sum_{j=0}^{n-1} \alpha^{j}}\right),\right. \\
\left.\mu_{\varphi(2 x, x)}^{\prime}\left(\frac{4^{m+1} t}{4^{m} \alpha^{m} 2 \sum_{j=0}^{n-1} \alpha^{j}}\right)\right) \\
=T_{k=1}^{n+1}\left(\mu_{4 \varphi(x, x)}^{\prime}\left(\frac{4 t}{2 \sum_{j=0}^{n+m-1} \alpha^{j}}\right),\right. \\
\left.\mu_{\varphi(2 x, x)}^{\prime}\left(\frac{4 t}{2 \sum_{j=0}^{n+m-1} \alpha^{j}}\right)\right)
\end{gathered}
$$

when $m, n$ tend to $\infty$. Then $\left\{g\left(2^{n} x\right) / 4^{n}\right\}$ is a Cauchy sequence in $(Y, \mu, T)$. Since $(Y, \mu, T)$ is a complete $\mathrm{RN}$-space this sequence converges to some point $Q(x) \in Y$. Fix $x \in X$ and put $m=0$ in (57). Thus

$$
\mu_{g\left(2^{n} x\right) / 4^{n}-g(x)}(t)
$$

$$
\begin{array}{r}
\geq T_{k=1}^{n+1}\left(\mu_{4 \varphi(x, x)}^{\prime}\left(\frac{4 t}{2 \sum_{j=0}^{n-1} \alpha^{j}}\right),\right. \\
\left.\mu_{\varphi(2 x, x)}^{\prime}\left(\frac{4 t}{2 \sum_{j=0}^{n-1} \alpha^{j}}\right)\right),
\end{array}
$$

for every $\delta, t>0, x \in X$, and from (58) we have

$$
\begin{aligned}
& \mu_{\mathrm{Q}(x)-g(x)}(t+\delta) \\
& =\mu_{\mathrm{Q}(x)-g\left(2^{n} x\right) / 4^{n}+g\left(2^{n} x\right) / 4^{n}-g(x)}(t+\delta) \\
& \geq T\left(\mu_{\mathrm{Q}(x)-g\left(2^{n} x\right) / 4^{n}}(\delta),\right. \\
& \left.\mu_{g\left(2^{n} x\right) / 4^{n}-g(x)}(t)\right) \\
& \geq T\left(\mu_{\mathrm{Q}(x)-g\left(2^{n} x\right) / 4^{n}}(\delta),\right. \\
& T_{k=1}^{n+1}\left(\mu_{4 \varphi(x, x)}^{\prime}\left(\frac{4 t}{2 \sum_{j=0}^{n-1} \alpha^{j}}\right),\right. \\
& \left.\left.\mu_{\varphi(2 x, x)}^{\prime}\left(\frac{4 t}{2 \sum_{j=0}^{n-1} \alpha^{j}}\right)\right)\right),
\end{aligned}
$$

we know when $n$ tend $\infty$, then $g\left(2^{n} x\right) / 4^{n} \rightarrow Q(x)$, and $\sum_{j=0}^{n-1} \alpha^{j} \rightarrow(1 /(1-\alpha))$. Therefore taking the $\lim n \rightarrow \infty$ and using (59), also by using Definitions 1(iii) and 2(RN2) we get

$$
\begin{gathered}
\mu_{\mathrm{Q}(x)-g(x)}(t+\delta) \\
\geq T\left(1, T_{k=1}^{\infty}\left(\mu_{4 \varphi(x, x)}^{\prime}\left(\frac{4(1-\alpha) t}{2}\right),\right.\right. \\
\left.\left.\mu_{\varphi(2 x, x)}^{\prime}\left(\frac{4(1-\alpha) t}{2}\right)\right)\right) \\
\geq T_{k=1}^{\infty}\left(\mu_{(1 / 2) \varphi(x, x)}^{\prime}\left(\frac{(1-\alpha) t}{2}\right),\right. \\
\left.\mu_{(1 / 4) \varphi(2 x, x)}^{\prime}\left(\frac{(1-\alpha) t}{2}\right)\right),
\end{gathered}
$$

since $\delta$ was arbitrary, and by taking $\delta \rightarrow 0$ in (60), we get

$$
\begin{array}{r}
\mu_{g(x)-\mathrm{Q}(x)}(t) \geq T_{k=1}^{\infty}\left(\mu_{(1 / 2) \varphi(x, x)}^{\prime}\left(\frac{(1-\alpha) t}{2}\right),\right. \\
\left.\mu_{(1 / 4) \varphi(2 x, x)}^{\prime}\left(\frac{(1-\alpha) t}{2}\right)\right),
\end{array}
$$

which implies that the Inequality (39) holds. Replacing $x$ and $y$ by $2^{n} x$ and $2^{n} y$ in (38), respectively, we get

$$
\mu_{f\left(2^{n} x+2^{n+1} y\right)+f\left(2^{n} x-2^{n+1} y\right)-4 f\left(2^{n} x+2^{n} y\right)-4 f\left(2^{n} x-2^{n} y\right)+6 f\left(2^{n} x\right)-2 f\left(2^{n+1} y\right)+8 f\left(2^{n} y\right)}(t) \geq \mu_{\varphi\left(2^{n} x, 2^{n} y\right)}^{\prime}(t)
$$


that by division by the $4^{n}$ we have

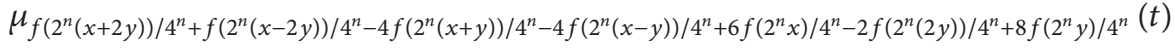

$$
\begin{aligned}
& \geq \mu_{\varphi\left(2^{n} x, 2^{n} y\right)}^{\prime}\left(4^{n} t\right) \geq \mu_{8^{n} \alpha^{n} \varphi(x, y)}^{\prime}\left(4^{n} t\right) \geq \mu_{\varphi(x, y)}^{\prime}\left(\frac{t}{\alpha^{n}}\right) \longrightarrow 1,
\end{aligned}
$$

for all $x, y \in X$ and for all $t>0$, then $Q$ fulfills (1). To prove the uniqueness of the quadratic function $Q$, let us assume that there exists a quadratic function $H: X \rightarrow Y$ which satisfies (39). Obviously we have $Q\left(2^{n} x\right)=4^{n} Q(x)$ and $H\left(2^{n} x\right)=$ $4^{n} H(x)$ for all $x \in X$. From (39) and (61) we have

$$
\begin{aligned}
\mu_{\mathrm{Q}(x)-H(x)}(t) \geq T\left(\mu_{\mathrm{Q}\left(2^{n} x\right)-g\left(2^{n} x\right)}\left(\frac{4^{n} t}{2}\right), \mu_{g\left(2^{n} x\right)-\mathrm{Q}\left(2^{n} x\right)}\left(\frac{4^{n} t}{2}\right)\right) \\
\geq T\left(1, T_{k=1}^{\infty}\left(\mu_{\varphi\left(2^{n} x, 2^{n} x\right)}^{\prime}\left((1-\alpha) \frac{4^{n} t}{2}\right),\right.\right. \\
\left.\left.\mu_{(1 / 4) \varphi\left(2^{n+1} x, 2^{n} x\right)}^{\prime}\left((1-\alpha) \frac{4^{n} t}{2}\right)\right)\right) \\
=T_{k=1}^{\infty}\left(\mu_{4^{n} \alpha^{n} \varphi(x, x)}^{\prime}\left((1-\alpha) \frac{4^{n} t}{2}\right),\right. \\
\geq T_{k=1}^{\infty}\left(\mu_{\varphi\left(4^{n} \alpha^{n} / 4\right) \varphi(2 x, x)}^{\prime}\left((1-\alpha) \frac{4^{n} t}{2}\right)\right) \\
\left.\mu_{(1 / 4) \varphi(2 x, x)}^{\prime}\left((1-\alpha) \frac{t}{2 \alpha^{n}}\right)\right) .
\end{aligned}
$$

Since $\lim _{n \rightarrow \infty}\left((1-\alpha)\left(t / 2 \alpha^{n}\right)\right)=\infty$, we get $\lim _{n \rightarrow \infty} \mu_{\varphi(x, x)}^{\prime}$ $\left((1-\alpha)\left(t / 2 \alpha^{n}\right)\right)=1$, and $\mu_{n \rightarrow \infty}^{\prime} \mu_{(1 / 4) \varphi(2 x, x)}\left((1-\alpha)\left(t / 2 \alpha^{n}\right)\right)=$ 1 , then $\mu_{\mathrm{Q}(x)-H(x)}(t)=1$, for all $t>0$. And so $Q(x)=H(x)$. This completes the proof.

\section{Conflict of Interests}

The authors declare that there is no conflict of interests regarding the publication of this paper.

\section{Acknowledgment}

The authors would like to thank the referee for giving useful comments and suggestions for the improvement of this paper.

\section{References}

[1] S. M. Ulam, Problems in Modern Mathematics, Science Editions John Wiley \& Sons, New York, NY, USA, 1964.

[2] D. H. Hyers, "On the stability of the linear functional equation," Proceedings of the National Academy of Sciences of the United States of America, vol. 27, pp. 222-224, 1941.
[3] T. Aoki, "On the stability of the linear transformation in Banach spaces," Journal of the Mathematical Society of Japan, vol. 2, pp. 64-66, 1950.

[4] T. M. Rassias, "On the stability of the linear mapping in Banach spaces," Proceedings of the American Mathematical Society, vol. 72, no. 2, pp. 297-300, 1978.

[5] C. Baak and M. S. Moslehian, "On the stability of $J^{*}$ homomorphisms," Nonlinear Analysis: Theory, Methods \& Applications, vol. 63, no. 1, pp. 42-48, 2005.

[6] S. Czerwik, Functional Equations and Inequalities in Several Variables, World Scientific, River Edge, NJ, USA, 2002.

[7] D. H. Hyers, G. Isac, and T. M. Rassias, Stability of Functional Equations in Several Variables, Birkhäauser, Boston, Mass, USA, 1998.

[8] S.-M. Jung, Hyers-Ulam-Rassias Stability of Functional Equations in Mathematical Analysis, Hadronic Press, Palm Harbor, Fla, USA, 2001.

[9] T. M. Rassias, Functional Equations, Inequalities and Applications, Kluwer Academic, Dodrecht, The Netherlands, 2003.

[10] R. P. Agarwal, Y. J. Cho, R. Saadati, and S. Wang, "Nonlinear 1 fuzzy stability of cubic functional equations," Journal of Inequalities and Applications, vol. 2012, article 77, 2012.

[11] J. M. Rassias, R. Saadati, G. Sadeghi, and J. Vahidi, "On nonlinear stability in various random normed spaces," Journal of Inequalities and Applications, vol. 2011, article 62, 2011.

[12] Y. J. Cho and R. Saadati, "Lattictic non-Archimedean random stability of ACQ functional equation," Advances in Difference Equations, p. 2011, article 31, 2011.

[13] D. Miheţ and R. Saadati, "On the stability of the additive Cauchy functional equation in random normed spaces," Applied Mathematics Letters, vol. 24, no. 12, pp. 2005-2009, 2011.

[14] C. Park, M. Eshaghi Gordji, and R. Saadati, "Random homomorphisms and random derivations in random normed algebras via fixed point method," Journal of Inequalities and Applications, vol. 2012, article 194, 2012.

[15] A. Ebadian, M. Eshaghi Gordji, H. Khodaei, R. Saadati, and Gh. Sadeghi, "On the stability of an $m$-variables functional equation in random normed spaces via fixed point method," Discrete Dynamics in Nature and Society, vol. 2012, Article ID 346561, 13 pages, 2012.

[16] J. I. Kang and R. Saadati, "Approximation of homomorphisms and derivations on non-Archimedean random Lie $C^{*}$-algebras via fixed point method," Journal of Inequalities and Applications, p. 2012, article 251, 2012.

[17] R. Saadati and S. M. Vaezpour, "Some results on fuzzy Banach spaces," Journal of Applied Mathematics \& Computing, vol. 17, no. 1-2, pp. 475-484, 2005.

[18] R. Saadati and C. Park, "Non-Archimedian $L$-fuzzy normed spaces and stability of functional equations," Computers \& Mathematics with Applications, vol. 60, no. 8, pp. 2488-2496, 2010. 
[19] S. J. Lee and R. Saadati, "On stability of functional inequalities at random lattice $\varphi$-normed spaces," Journal of Computational Analysis and Applications, vol. 15, no. 8, pp. 1403-1412, 2013.

[20] J. Vahidi, C. Park, and R. Saadati, "A functional equation related to inner product spaces in non-Archimedean $L$-random normed spaces," Journal of Inequalities and Applications, vol. 2012, article 168, 2012.

[21] Y. J. Cho and R. Saadati, "Lattictic non-Archimedean random stability of ACQ functional equation," Advances in Difference Equations, vol. 2011, article 31, 2011.

[22] D. Miheţ and V. Radu, "On the stability of the additive Cauchy functional equation in random normed spaces," Journal of Mathematical Analysis and Applications, vol. 343, no. 1, pp. 567$572,2008$.

[23] F. Skof, "Local properties and approximation of operators," Rendiconti del Seminario Matematico e Fisico di Milano, vol. 53, pp. 113-129, 1983.

[24] B. Schweizer and A. Sklar, Probabilistic Metric Spaces, NorthHolland, New York, NY, USA, 1983.

[25] A. N. Šerstnev, "On the concept of a stochastic normalized space," Doklady Akademii Nauk SSSR, vol. 149, pp. 280-283, 1963 (Russian). 


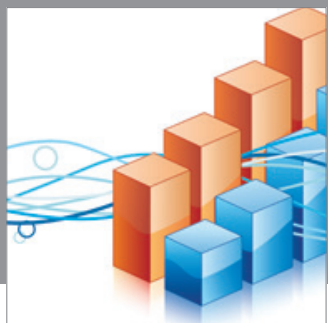

Advances in

Operations Research

mansans

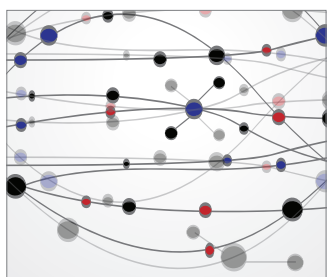

The Scientific World Journal
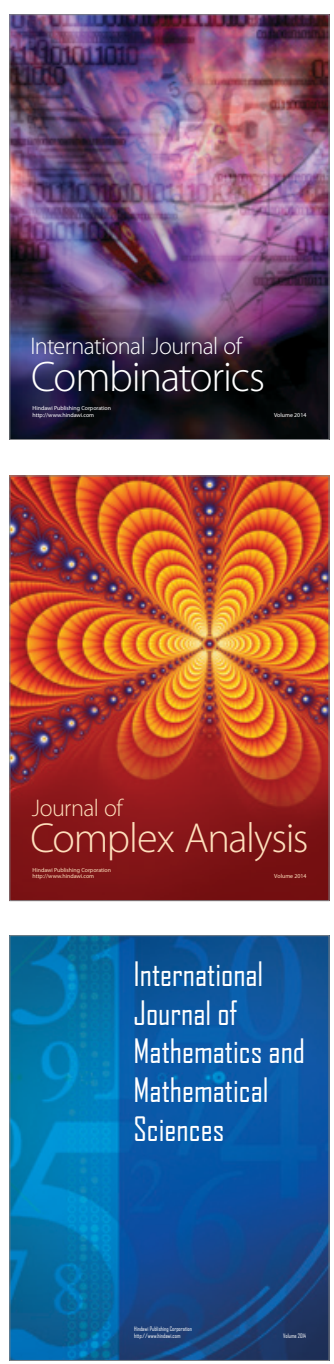
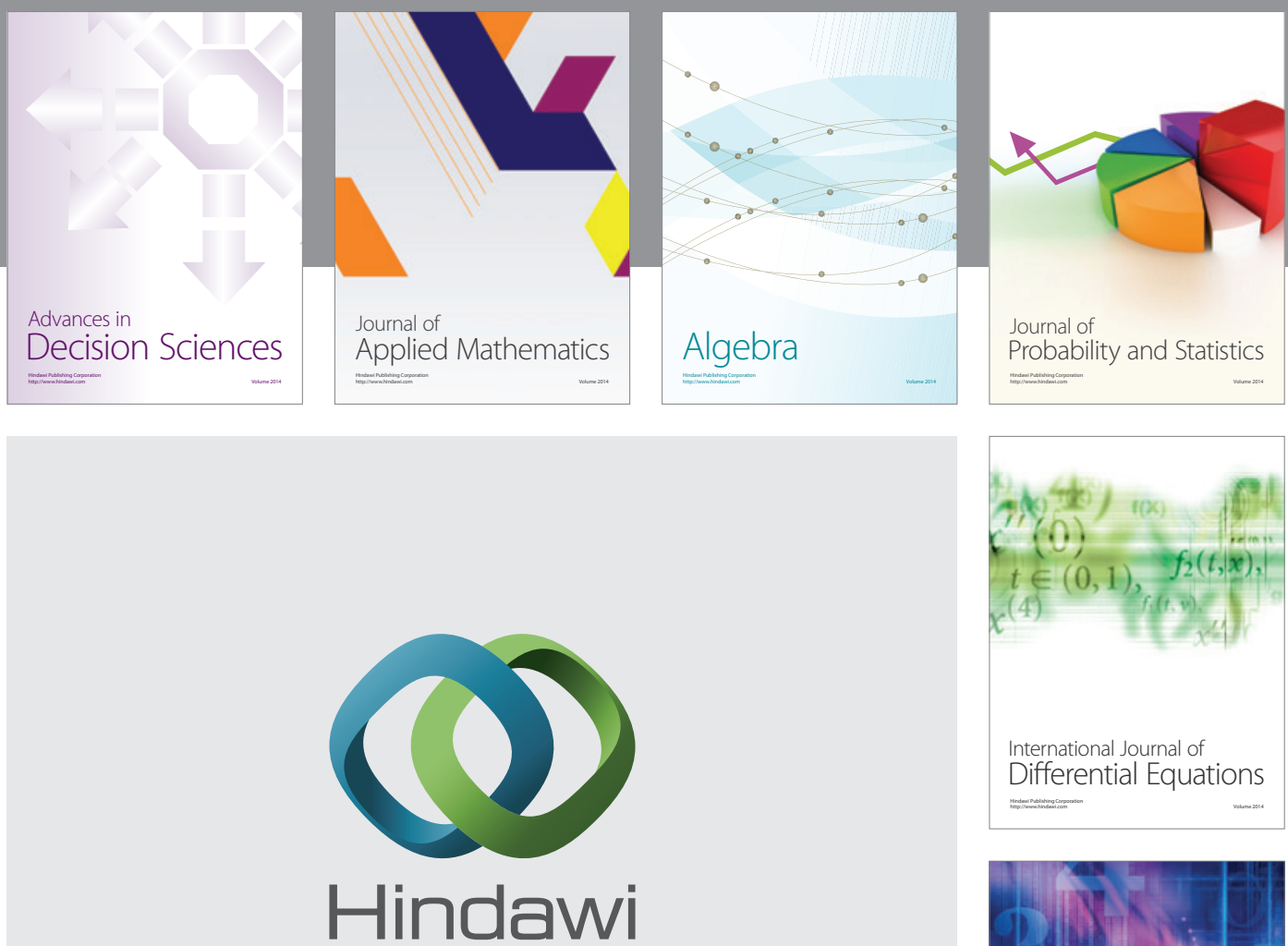

Submit your manuscripts at http://www.hindawi.com
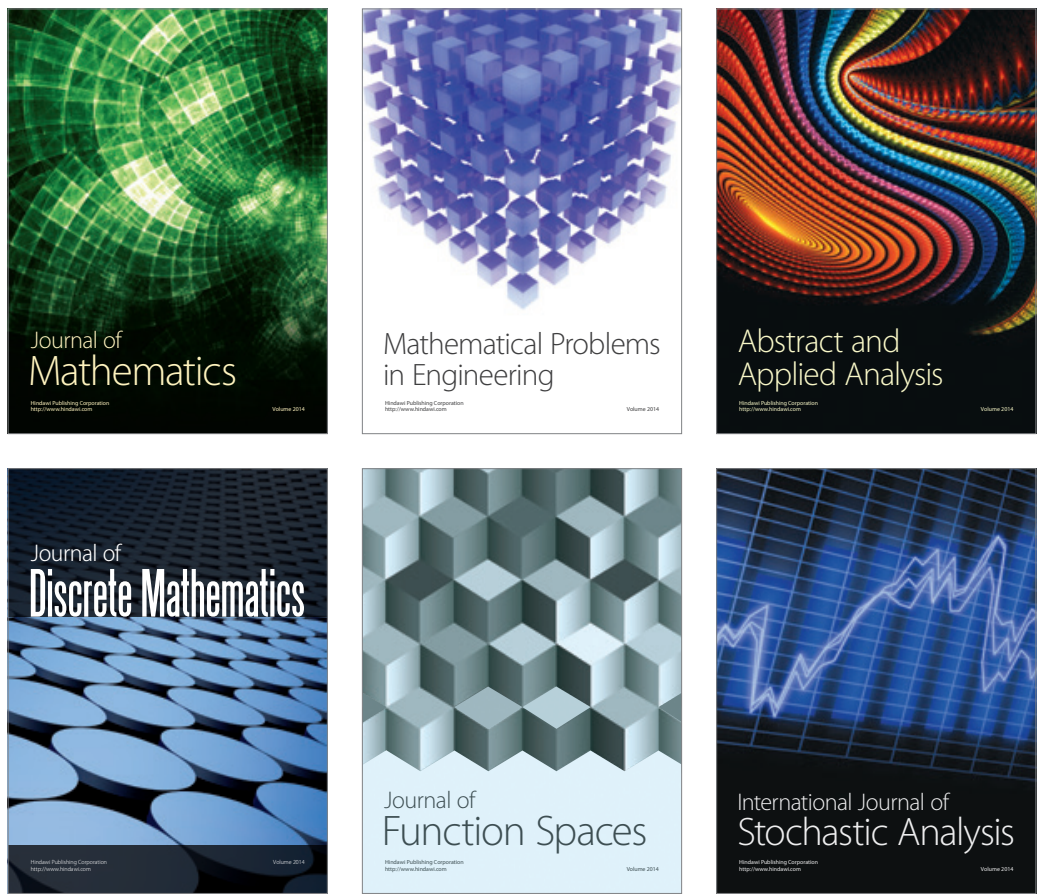

Journal of

Function Spaces

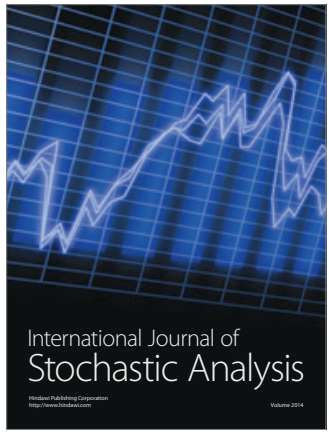

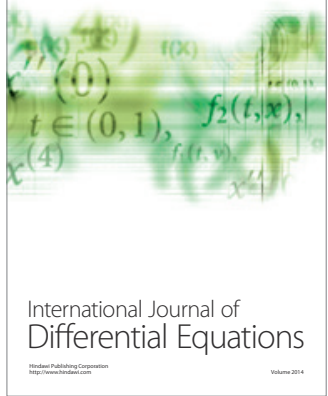
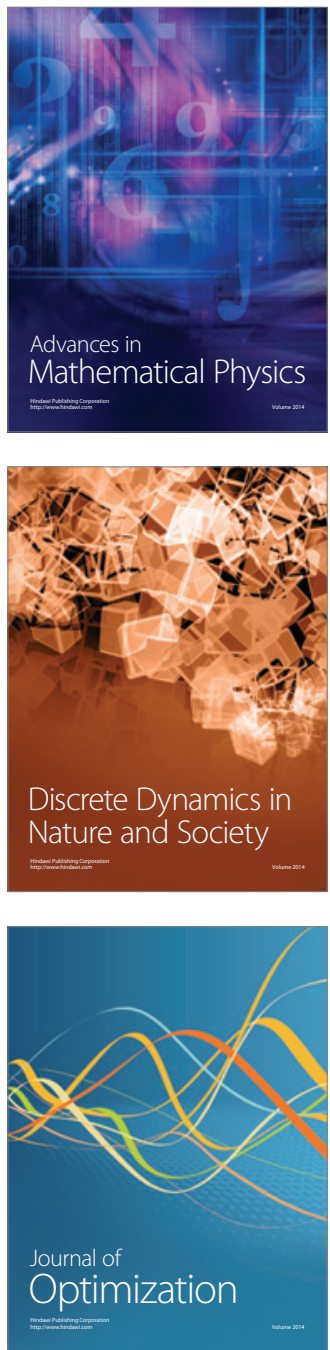